\title{
A PREVALÊNCIA DE HIPERTENSÃO ARTERIAL EM IDOSOS ATENDIDOS NO CENTRO DE CONVIVÊNCIA PARA IDOSOS EM CUIABÁ
}

\author{
Roberto Gomes de Azevedo* \\ Miguel Angel Claros Paz ${ }^{* *}$
}

\section{Resumo}

Esse estudo teve como objetivo principal verificar se a prevalência de hipertensão arterial essencial encontrada entre os idosos atendidos no Centro de Convivência para Idosos de Cuiabá-MT, na região Centro Oeste do País, no mês de outubro de 2003, seria superior a $50 \%$, que é o percentual de prevalência encontrado na literatura. Para a coleta de dados foi elaborado um questionário orientado abrangendo todas as variáveis: idade, hábito alimentar, sedentarismo, raça, obesidade, etilismo, tabagismo e o motivo pelo qual procurou a instituição. Foi um estudo de levantamento quantitativo, transversal, observacional e descritivo, de prevalência, no qual foram utilizados o raciocínio indutivo e a freqüência relativa como procedimento estatístico na apresentação e discussão dos resultados. A população amostral foi constituída pelos idosos que freqüentaram a instituição no mês de outubro de 2003. Os dados foram apresentados através de tabelas simples. Na discussão abordouse os fatores de risco como idade, sexo, alimentação dietética, cor da pele, sedentarismo, tabagismo e alcoolismo, comparando-se os dados da literatura com os achados na amostra. Concluiu-se que a incidência de hipertensão arterial essencial na população estudada foi superior a $50 \%$, atinge principalmente homens, pessoas da raça negra e sedentários.

Palavras-chave: Idosos. Hipertensão. Fatores de Risco.

* Médico Pós-Graduado em Geriatria e Gerontologia, Título de Especialista em Cirurgia Geral pela Associação Médica Brasileira - AMB. E-mail:robertoga@terra.com.br

** Médico Pós Graduando em Cardiologia; Pós Graduado em Ginecologia e Obstetrícia; Especialização em Saúde Pública e Pós Graduado em Geriatria. 


\section{Introdução}

O fenômeno do envelhecimento populacional, vivenciado por países desenvolvidos e em desenvolvimento, desperta a necessidade de pesquisas sobre idosos. Junto com o fenômeno do envelhecimento populacional, ocorre o aumento na prevalência de doenças crônico degenerativas associadas à idade (HAYFLICK, 1994). Segundo Lopes e Guimarães (2006) a hipertensão arterial essencial apresenta alta prevalência mundial e baixa porcentagem de controle com tratamento (em torno de 30\% nos Estados Unidos da América - EUA). No Brasil varia de 22 a 44\% dependendo da região considerada. Considerando ainda que muitos pacientes desconhecem ter a doença e, dos que sabem, $40 \%$ estão sem tratamento.

No ano de 2003, no Brasil, segundo Lopes e Guimarães (2006), ocorreram um milhão de óbitos, sendo que destes, $28 \%$ decorrentes de doenças cardiovasculares. Dentre os fatores de risco cardiovasculares para a mortalidade, a hipertensão arterial está associada a $40 \%$ das mortes por acidente vascular encefálico e a $25 \%$ por doença coronária.

Estudos epidemiológicos brasileiros demonstram que a prevalência de hipertensão arterial entre idosos, à semelhança da observada em todo o mundo, é bastante elevada. Cerca de $65 \%$ dos idosos são hipertensos, e entre as mulheres com mais de 75 anos a prevalência de hipertensão pode chegar a 80\% (LESSA, 1998).

É provável que grande número de fatores inter-relacionados contribuam para a alteração da pressão arterial no idoso. Entre os fatores mais estudados estão a ingestão de sal, obesidade e a vida sedentária, sendo que nos últimos anos passou-se a valorizar o fator genético (LORGA et al., 2002).

A prevalência de hipertensão arterial essencial nos Estados Unidos foi em torno de $60 \%$ entre os brancos e de $71 \%$ entre os negros com idade acima de 60 anos. No centro-oeste brasileiro a prevalência varia entre 6,3\% e 16,75\%, independentemente da raça (AMODEO, 1997).

Podemos enunciar que a hipertensão arterial essencial também é freqüente no Centro de Convivência de Idosos Cuiabá - MT, assim como em qualquer outra unidade recreativa ou de assistência à saúde dos idosos.

Orsine Valente (2006) afirma que uma variedade de modificações dietéticas e no estilo de vida são benéficas no tratamento da hipertensão, incluindo restrição de sal, redução do peso e possivelmente aumento da 
ingestão de potássio e cálcio, além de dieta vegetariana ou suplementos de óleo de peixe.

No Centro de Convivência para Idosos de Cuiabá - MT, tem-se observado uma alta freqüência no uso de medicação anti-hipertensiva, o que suscitou o estudo para o levantamento da prevalência da hipertensão arterial essencial nesta população no mês de outubro de 2003. Devido a isto, a fim de prevenir e detectar os déficits advindos do envelhecimento, o objetivo deste estudo foi pesquisar se a prevalência da hipertensão arterial essencial na instituição em estudo era superior a $16,75 \%$, que é o percentual encontrado no presente estudo e na literatura nacional, como índice de prevalência na população de idosos.

\section{Material e Método}

Este é um estudo quantitativo, transversal, observacional e descritivo, um estudo de ocorrência de prevalência, no qual foram utilizados o raciocínio indutivo e os procedimentos estatísticos na apresentação e discussão dos resultados. Fizeram parte da população desse estudo, os idosos que freqüentaram o Centro de Convivência para Idosos de Cuiabá - MT no mês de outubro de 2003. Foram avaliados 600 pacientes que procuraram a instituição. Para a coleta de dados foi elaborado um questionário orientado abrangendo as variáveis deste estudo. Os dados foram apresentados através de tabelas. A discussão foi baseada na literatura e na reflexão sobre os achados.

\subsection{Definição de Termos}

Tavares e Ribeiro (2006, p. 6), asseguram que a “[ . . . ] definição operacional de hipertensão arterial poderia ser entendida como sendo o estado hipertensivo no qual a ação de tratar ultrapassa os benefícios da ação de não tratar." Como parâmetros foram utilizados os critérios das IV Diretrizes Brasileiras - 2004 (MION JÚNIOR et al., 2004, online). 
TABELA 1 - Critérios das IV Diretrizes Brasileiras - 2004

\begin{tabular}{l|l|l}
\hline C łassificação & $\begin{array}{l}\text { P ressão } \\
\text { S is tólica } \\
(\mathrm{m} \mathrm{m} \mathrm{H} \mathrm{g})\end{array}$ & $\begin{array}{l}\text { P ressão } \\
\text { D iastólica } \\
(\mathrm{m} \mathrm{m} \mathrm{H} \mathrm{g})\end{array}$ \\
\hline Ó tin a & $<120$ & $<80$ \\
\hline Norm al & $<130$ & $<85$ \\
\hline Lin trofe & $130-139$ & $85-89$ \\
\hline
\end{tabular}

H p̣ertensão

\begin{tabular}{l|l|l}
\hline Estági I ( leve) & $140-159$ & $90-99$ \\
\hline $\begin{array}{l}\text { Estági II } \\
\text { ( moderada) }\end{array}$ & $160-179$ & $100-109$ \\
\hline $\begin{array}{l}\text { Estági III } \\
\text { ( grave) }\end{array}$ & $>180$ & $>110$ \\
\hline Sistólica isolada & $>140$ & $<90$ \\
\hline
\end{tabular}

\subsection{Metodologia de Aferição da Pressão Arterial}

$\mathrm{Na}$ fase de preparo do paciente: o procedimento é explicado, deixando-o previamente por cerca de cinco minutos em ambiente calmo, solicitando que o paciente esvazie a bexiga previamente, que não tenha praticado exercícios físicos nos últimos 90 minutos; que não tenha ingerido bebidas alcoólicas, café, fumado ou alimentado-se 30 minutos antes; mantendo o paciente deitado, aferindo a pressão no braço direito de todos os avaliados, mantendo as pernas descruzadas, pés apoiados na mesa de exame; o manguito é colocado no braço desnudo, sendo este braço posicionado ao nível do coração (nível do $4^{\circ}$ espaço Intercostal), com a palma da mão voltada para cima e cotovelo ligeiramente fletido; recomendando ainda que durante o processo de aferição o paciente não fale e nem provoque tosse. 
No procedimento de medida da pressão arterial: o manguito é selecionado de acordo com o perímetro do braço, com o bordo inferior do manguito a mais ou menos $3 \mathrm{~cm}$ da fossa cubital. Palpa-se o pulso da artéria braquial, coloca-se a campânula do estetoscópio sobre esse ponto, sem compressão; infla-se até a pressão do manômetro ultrapassar $40 \mathrm{mmHg}$ da pressão aferida digitalmente; procede-se a deflação na velocidade de 2 a 4 $\mathrm{mmHg}$ por segundo. A pressão sistólica foi considerada no primeiro som (fase I de Korotkoff). A pressão diastólica foi considerada no desaparecimento do som (fase V Korotkoff); em todos os pacientes foi realizada uma segunda medida cerca de 3 minutos após. Todos foram informados dos valores aferidos (MION JÚNIOR; NOBRE, 1997; III CONSENSO BRASILEIRO DE CARDIOLOGIA, 1998).

Aparelho: foram utilizados esfigmanômetros da marca Taykos, calibrado pelo Instituto Nacional de Metrologia (INMETRO) em julho de 2003, com manguitos de dois tamanhos.

Variáveis: motivo da consulta, sexo, raça, praticantes de atividade física, alimentação dietética, tabagismo ativo; alcoolismo ativo; motivo da procura do Centro de Convivências para Idosos.

Critério de idoso: pacientes com mais de 60 anos e cadastrados no Centro de Convivências para Idosos.

Critérios de inclusão: considerou-se portador de hipertensão arterial sistêmica todo paciente com pressão arterial 140 x $90 \mathrm{mmHg}$; foram incluídos todos os pacientes com mais de 60 anos de idade que procuraram o Centro de Convivência para Idosos de Cuiabá - MT no mês de outubro de 2003; foram incluídos os novos pacientes que procuraram a instituição pela primeira vez e aqueles em tratamento crônico. Foram incluídos na amostra os casos de hipertensão arterial essencial.

Critérios de exclusão: naqueles pacientes nos quais suspeitou-se da hipertensão "do avental branco", que não tinha lesão em órgão alvo, fatores de risco cardiovasculares e sem uso de drogas anti-hipertensivas. Todos os pacientes com menos de 60 anos e os com pressão arterial normal.

Foram excluídos os casos de hipertensão secundária, baseados em critérios clínicos e da medicina baseada em evidencias: inicio antes dos 30 anos ou após os 50 anos; hipertensão resistente ao tratamento; uso de drogas que elevam a pressão arterial; fácies ou biótipo de Doença Renal, Síndrome de Cushing, hipertiroidismo, acromegalia; massas ou sopros abdominais; 
assimetria de pulsos; doença arterial isquêmica prévia; hipertensão arterial maligna.

Cálculo estatístico: foi utilizada somente a freqüência relativa como tratamento dos dados coletados.

\section{Resultados}

Foram investigados 600 (seiscentos) idosos que freqüentaram o Centro de Convivência para Idosos de Cuiabá-MT, no mês de outubro de 2003.

TABELA 2 - Determinação da freqüência relativa da prevalência de hipertensão arterial sistólica, quanto ao motivo da consulta, dos pacientes atendidos no ambulatório do Centro de Convivência para Idosos, no mês de outubro de 2003

\begin{tabular}{l|l|l}
\hline M otivo da C onsulta & N & $\%$ \\
\hline $\begin{array}{l}\text { H piertensão Arteríl } \\
\text { S istólica }\end{array}$ & 341 & 56,8 \\
\hline Outros & 259 & 43,2 \\
\hline Total & 600 & 100 \\
\hline
\end{tabular}

A Tabela 2 mostra que a prevalência de hipertensão foi maior que $56 \%$ da população atendida, conforme dados da literatura. Para Antonio Fernandes Moron (2006), referindo-se a estudos epidemiológicos brasileiros sobre hipertensão, a prevalência oscila de 22 a $44 \%$ na população adulta, chegando a $55 \%$ entre aqueles com mais de 50 anos de idade.

TABELA 3 - Determinação da freqüência relativa da prevalência de hipertensão arterial sistólica, quanto ao sexo, dos pacientes atendidos no ambulatório do Centro de Convivência para Idosos, no mês de outubro de 2003

\begin{tabular}{l|l|l}
\hline Sexo & N & $\%$ \\
\hline Masculino & 249 & 73,0 \\
\hline Fem inino & 92 & 27,0 \\
\hline Total & 341 & 100 \\
\hline
\end{tabular}


A Tabela 3 evidencia que dois terços dos idosos hipertensos são do sexo masculino. Ramos e Brito (2006), referindo aos fatores de risco que influenciam o prognóstico da hipertensão arterial no idoso, proposto pela Organização Mundial da Saúde, em 1999, consideram a idade acima de 55 anos para os homens e 65 anos para as mulheres como importante fator de risco para o desenvolvimento de doença cardiovascular. Portanto, para os homens, o risco é maior em idade menor, quando comparado com as mulheres.

TABELA 4 - Determinação da freqüência relativa da prevalência de hipertensão arterial sistólica, quanto a raça, dos pacientes atendidos no ambulatório do Centro de Convivência para Idosos, no mês de outubro de 2003

\begin{tabular}{l|l|l}
\hline R aça & N & $\%$ \\
\hline Negra & 232 & 68,0 \\
\hline Branca & 109 & 32,0 \\
\hline Total & 341 & 100 \\
\hline
\end{tabular}

A Tabela 4 evidencia que a maioria dos idosos hipertensos em estudo é da raça negra. Para Moffa e Sanches (2001) a hipertensão (essencial) primária ocorre em $95 \%$ dos casos, nenhuma causa pode ser estabelecida. Essa condição ocorre em 10-15 \% dos adultos de raça branca e em 20$30 \%$ dos adultos de raça negra nos Estados Unidos.

TABELA 5 - Determinação da freqüência relativa da prevalência de hipertensão arterial sistólica, quanto à atividade física, dos pacientes atendidos no ambulatório do Centro de Convivência para Idosos, no mês de outubro de 2003

\begin{tabular}{l|l|l}
\hline $\begin{array}{l}\text { P rática de } \\
\text { A tividade Física }\end{array}$ & N & $\%$ \\
\hline Sin & 66 & 11,0 \\
\hline Não & 534 & 89,0 \\
\hline Total & 600 & 100 \\
\hline
\end{tabular}


A Tabela 5 mostra que a maior parte dos idosos em estudo (89\%) não apresentavam hábito de exercícios físicos para prevenção da hipertensão arterial. Orsine Valente (2006) referindo-se à associação entre exercício físico e a hipertensão, afirma que o exercício aeróbico tem efeito benéfico no controle da pressão arterial sistólica. O exercício de moderada intensidade por 30 minutos cinco a sete vezes por semana por 12 semanas, resultou em aumentada produção de óxido nítrico com conseqüente vasodilatação endotelial e queda na pressão arterial.

TABELA 6 - Determinação da freqüência relativa da prevalência de hipertensão arterial sistólica, quanto à alimentação dietética, dos pacientes atendidos no ambulatório do Centro de Convivência para Idosos, no mês de outubro de 2003

\begin{tabular}{l|l|l}
\hline $\begin{array}{l}\text { A lim entação } \\
\text { D ietética }\end{array}$ & N & $\%$ \\
\hline S in & 72 & 12,0 \\
\hline Não & 528 & 88,0 \\
\hline Total & 600 & 100 \\
\hline
\end{tabular}

A Tabela 6 mostra que os idosos em estudo não se preocupam com cuidados alimentares como medidas preventivas. Orsine Valente (2006) assevera que a restrição de sódio aumenta a eficácia de diversos antihipertensivos, diminui a pressão arterial e o grau de depleção de potássio durante o tratamento com diurético. Os negros são mais sensíveis à restrição de sódio do que os brancos, devido a menor responsividade do sistema renina angiotensina. Uma dieta muito baixa em sódio pode produzir efeitos cardiovasculares adversos, mas a maioria dos autores atuais evidencia a segurança e eficácia da restrição moderada de sódio.

TABELA 7 - Determinação da freqüência relativa da prevalência de hipertensão arterial sistólica, quanto ao tabagismo ativo, dos pacientes atendidos no ambulatório do Centro de Convivência para Idosos, no mês de outubro de 2003

\begin{tabular}{l|l|l}
\hline Tabagism o & N & $\%$ \\
\hline S in & 40 & 11,7 \\
\hline Não & 301 & 88,3 \\
\hline Total & 341 & 100 \\
\hline
\end{tabular}


A Tabela 7 mostra que a incidência de tabagismo é relativamente baixa na população em estudo. Orsine Valente (2006) assegura que o hábito de fumar pode produzir aumento transitório na pressão arterial de aproximadamente 5 a $10 \mathrm{mmHg}$. Esse efeito pode ser mais proeminente com o primeiro cigarro em fumantes habituais. A pressão arterial aumenta durante o dia quando o paciente está fumando, mas retorna ao basal quando o paciente esta dormindo ou está no consultório. Nos pacientes idosos o efeito da nicotina se soma ao efeito da vasoconstrição, aumentando ainda mais a pressão e acentua a doença vascular de base presente nos pacientes idosos.

TABELA 8 - Determinação da freqüência relativa da prevalência de hipertensão arterial sistólica, quanto ao alcoolismo, dos pacientes atendidos no ambulatório do Centro de Convivência para Idosos, no mês de outubro de 2003

\begin{tabular}{l|l|l}
\hline A lcoolism o & N & $\%$ \\
\hline S in & 46 & 13,5 \\
\hline Não & 295 & 86,5 \\
\hline Total & 341 & 100 \\
\hline
\end{tabular}

A Tabela 8 evidencia que o consumo de álcool, mesmo que seja um agravante para a hipertensão no idoso, apresenta baixa incidência na população em estudo. Moffa e Sanches (2001), afirmam que o uso excessivo de álcool também aumenta a pressão arterial, talvez através do aumento das catecolaminas plasmáticas, tornando difícil o controle da hipertensão em pacientes que consomem mais de $40 \mathrm{~g}$ de etanol (dois drinques) diariamente ou "tomam bebedeiras". 
TABELA 9 - Determinação da freqüência relativa da prevalência de hipertensão arterial sistólica, quanto ao objetivo da procura ao serviço, dos pacientes atendidos no ambulatório do Centro de Convivência para Idosos, no mês de outubro de 2003

\begin{tabular}{l|l|l}
\hline o bjetivo & N & $\%$ \\
\hline $\begin{array}{l}\text { Para controłra } \\
\text { hịertensão }\end{array}$ & 120 & 20,0 \\
\hline $\begin{array}{l}\text { Para pegar } \\
\text { m edicam entos }\end{array}$ & 90 & 15,0 \\
\hline $\begin{array}{l}\text { Para controhra } \\
\text { PA e pegar } \\
\text { m edicam entos }\end{array}$ & 360 & 60,0 \\
\hline $\begin{array}{l}\text { Não soube } \\
\text { responder }\end{array}$ & 30 & 5,0 \\
\hline Total & 600 & 100 \\
\hline
\end{tabular}

A Tabela 9 evidencia que o motivo mais alegado aplica-se ao interesse desta população amostral é para controlar a pressão arterial e pegar medicamentos. Totalizando $60 \%$ dos que foram atendidos no Centro de Convivências.

\section{Discussão dos Resultados}

Os dados apresentados mostram que a prevalência de hipertensão se encontra maior que $50 \%$ da população atendida, comprovando os dados da literatura (Tabela 2 e 3 ). Há evidência de que a maioria dos idosos hipertensos é do sexo masculino (Tabela 3), para Ramos e Brito (2006) afirmam que nos homens o início da hipertensão ocorre cerca de 10 anos antes quando comparado com as mulheres. A maioria dos idosos hipertensos em estudo é da raça negra. (Tabela 4), sendo que Moffa e Sanches (2001) afirmam que os negros e idosos tendem a apresentar atividade de renina plasmática mais baixa. Estes pacientes podem ter maiores volumes intravasculares. Estudos demonstram que a hipertensão na raça negra possui prevalência maior, pior evolução e complicações mais graves e freqüentes. Este aspecto talvez tenha correlação com o nível sócio-econômico deste grupo de pacientes, não raramente pertencentes a classes sociais mais baixas. 
No entanto, a função renal entra em declínio mais rapidamente neste grupo mesmo com controle eficaz da PA (GUYTON, 1998). A grande maioria dos idosos deste estudo (89\%) não apresentava hábito de exercícios físicos para prevenção da hipertensão arterial (Tabela 5). Moffa e Sanches (2001) afirmam que a relação entre o exercício e a hipertensão ainda é controversa. Asseguram, ainda, que o exercício aeróbio parece diminuir a pressão arterial em indivíduos previamente sedentários, e em pessoas anteriormente ativas, com exercícios progressivamente vigorosos, podem ter menor efeito. No mesmo sentido Orsine Valente (2006), ressaltando que as drogas anti-hipertensivas possam diminuir o desempenho ao exercício. Mesmo assim, dentre as medidas não farmacológicas de tratamento para hipertensão arterial sistêmica, a atividade física tem sido amplamente recomendada. Deve-se atentar para a adequação do treinamento físico para essa finalidade, ou seja, quais as características do treinamento físico que ampliam seu efeito hipotensor. Entre os hipertensos tem sido demonstrado que o treinamento físico diminui significativamente a pressão arterial de repouso, em média na ordem de $10 \mathrm{mmHg}$, tanto da pressão sistólica quanto da diastólica, nos casos de hipertensão leve (COTRAN; KUMAR; COLLINS, 2000).

Os idosos em estudo não se preocupam com cuidados alimentares como medidas preventivas. (Tabela 6). A Obesidade é uma condição crônica que aumenta a morbidade de muitas doenças e a mortalidade por todas as causas sendo considerada um dos maiores fatores de risco para doença arterial coronariana, além da associação com hipertensão arterial, diabetes mellitus e dislipidemia. A associação entre hipertensão e obesidade é reconhecida há muito tempo. Orsine Valente (2006) afirma que o mecanismo pelo qual a obesidade aumenta a pressão arterial ainda não é bem entendido. Acredita este autor, que há uma provável hiperinsulinemia causada pela obesidade $\mathrm{e}$ que poderia aumentar a pressão arterial em decorrência do aumento da atividade simpática, do aumento da reabsorção e por causar disfunção endotelial. Estudos populacionais mostram que a hipertensão dobra em adultos jovens e é pelo menos 50\% maior nos adultos mais velhos ( $40 \mathrm{a} 64$ anos) com excesso de peso, quando comparados com indivíduos com peso normal. Estes mesmos estudos mostram que excesso de peso aumenta em oito vezes a incidência de hipertensão, sendo que para cada quilo ganho a pressão sistólica aumenta em 1mmHg (COTRAN; KUMAR; COLLINS, 2000). No Brasil, e no mundo, está ocorrendo um aumento alarmante da população obesa, em ambos os sexos, faixas etárias e classes sociais. Além disso, contribui o 
envelhecimento populacional, a ingestão de alimentos industrializados, o aumento no consumo de refrigerantes e álcool, a redução de atividades físicas e o uso constante e crescente do automóvel. A hipertensão arterial, como qualquer doença, depende a interação das características genéticas próprias do paciente com seu meio ambiente. $\mathrm{O}$ fator genético tem forte influência sobre a hipertensão arterial primária. A hipertensão é, por conceito, uma doença poligênica e multifatorial. Tavares e Ribeiro (2006) afirmam que, entre os fatores conhecidos no desenvolvimento de hipertensão, o sistema nervoso simpático é o primeiro mediador nas mudanças súbitas ou agudas da pressão arterial, agindo sobre a vasoconstrição e no débito cardíaco, através da via baroreflexa. Outro fator envolvido é a sensibilidade ao sal, sendo que a pressão arterial em alguns indivíduos é responsiva ou "sensitiva" a variação de sal na dieta, enquanto em outros tal variação tem pouco ou nenhum efeito. A obesidade e a hipertensão arterial, independentemente, aumentam o risco cardiovascular. A resistência à insulina é fator de risco independente para o desenvolvimento da hipertensão arterial e doença cardiovascular. A hipertensão e a obesidade abdominal, fazem parte de uma constelação de fatores de risco cardiovasculares chamada Síndrome Metabólica. Outros fatores podem ser incluídos: diabete, dislipidemia com baixo nível de HDL e alto nível de triglicerídios com moléculas pequenas e densas de lipoproteínas de baixa densidade e altamente aterogênicas, microalbuminúria e anormalidades prótrombóticas. A Síndrome Metabólica acomete hoje 20\% da população americana. A prevalência de tabagismo ativo encontrado no estudo é relativamente baixa na população amostral (Tabela 7). Em relação ao consumo de álcool, mesmo que seja um agravante para a hipertensão no idoso, quando ingerido em grandes volumes e de forma repetida, apresenta baixa prevalência na população em estudo (Tabela 8). Há grande interesse da população estudada em freqüentar o Centro de Convivências para Idosos, sendo o principal motivo de dirigir-se ao local é o de controlar a pressão arterial e pegar medicamentos. Totalizando 60\% (Tabela 9).

\section{Conclusões}

A prevalência de hipertensão arterial entre os idosos foi superior a $50 \%$ dos casos estudados. Entre os fatores de risco, analisados e associados ao desenvolvimento da hipertensão arterial, ficaram demonstrado ocorrer mais em homens, em pessoas da raça negra, que não praticam exercícios 
físicos de forma regular. Não se mostrou importante o tabagismo e o alcoolismo nesta amostra, como fatores de risco à hipertensão arterial. $O$ principal fator que motiva os pacientes a se deslocarem até o Centro de Convivência de Idosos de Cuiabá é a aferição da pressão arterial e obter o medicamento de uso crônico.

\title{
THE PREVALENCE ARTERIAL HYPERTENSION IN OLD-AGE PEOPLE WHO ATTENDED AT "CENTRO DE CONVIVÊNCIA DE IDOSOS DE CUIABÁ - MATO GROSSO
}

\begin{abstract}
This study had as great objective to examine if the prevalent of essential arterial hypertension is found in between the old-aged people who attended at "Centro de Convivência de Idosos de Cuiabá - Mato Grosso, in October, 2003, was higher than $50 \%$, the prevalence found in the literature. In the survey it was done a direct questionnaire containing all the variables such as age, alimentary custom, sedentary life, race, obesity, ethyl and tobacco use, and reason to go there at the establishment. It was screening qualitative study, transversal, observational and describle of prevalence, where it was used the inductive judgment and realtive frequence as the statistic proceeding in the presentation and discussion of results. In this study part of population oldaged who visited the stabilishment, in October, 2003 was examined. The data detected was presented in singles charts. The discussion was based in risc factors as age, sex, customs alimentary, color of skin, sedentarism, tobacco and ethyl use, compared with data of the literature and in the discussion about the amostral data. It was conclude that the incidence of essential arterial hypertension in the population studied was higher than $50 \%$, reach mens, black race and sedent sedentary person.

Keywords: Arterial Hypertension. Old-Age. Risk Factors.
\end{abstract}




\section{REFERÊNCIAS}

III CONSENSO BRASILEIRO DE HIPERTENSÃO ARTERIAL. São Paulo: BG Cultural, 1998.

AMODEO, Celso; LIMA, Eliudem Galvão; VAZQUEZ, Elisardo C. Hipertensão Arterial. São Paulo: Sarvier, 1997.

COTRAN, Ramzi S.; KUMAR, Vinay; COLLINS, Stanley L. Robbins : Patologia estrutural e funcional. 6. ed. Rio de Janeiro: Guanabara Koogan, 2000.

GUYTON, Arthur C; HALL, John E. Fisiologia Humana e Mecanismos das Doenças. 6. ed. Rio de Janeiro: Guanabara Koogan, 1998.

HAYFLICK, Leonard. How and Why We Age. New York: Ballantine Books, 1994.

LESSA, Inês. O Adulto Brasileiro e as Doenças da Modernidade: epidemiologia das doenças crônicas não transmissíveis. São Paulo: Hucitec, 1998.

LOPES, Renato Delascio; GUIMARÃES, Hélio Penna. Avaliação Clínica do Paciente Hipertenso. SBM-Seminários Brasileiros em Medicina, São Paulo, v. 1, n. 1, p. 10-13, 2006.

LORGA, Adalberto et al. I Diretrizes do Grupo de Estudos em Cardiogeriatria da Sociedade Brasileira de Cardiologia. Arquivos Brasileiros de Cardiologia, São Paulo, v. 79, p. 1-40, 2002.

MION JÙNIOR, Décio; NOBRE, Fernando. Medida da Pressão Arterial: da teoria à prática. São Paulo: Lemos, 1997.

MION JÚNIOR, Decio et al. IV Brazilian Guidelines on Arterial Hypertension. Arquivos Brasileiros de Cardiologia, São Paulo, v. 82, p. 7-22, 2004. Suplemento 4. 
MOFFA, Paulo Jorge; SANCHES, Paulo César R. Hipertensão Sistêmica. In: TIERNEY JÚNIOR, Lawrence M.; MCPHEE, Stephen J.; PAPADAKIS, Maxime A. Diagnóstico \& Tratamento 2001. São Paulo: Atheneu, 2001. Cap. 11.

MORON, Antonio Fernandes. Editorial: hipertensão arterial. SBM Seminários Brasileiros em Medicina, São Paulo, v. 1, n. 1, p. 4, 2006.

RAMOS, Luiz Roberto; BRITO, Francisco Carlos de. Hipertensão Arterial no Idoso. SBM - Seminários Brasileiros em Medicina, São Paulo, v. 1, n. 1, p. 41-48, 2006.

TAVARES, Agostinho; RIBEIRO, Artur Beltrame. Definição e Fisiopatologia da Hipertensão Arterial. SBM-Seminários Brasileiros em Medicina, São Paulo, v. 1, n. 1, p. 5-9, 2006.

VALENTE, Orsine. Tratamento Não Farmacológico da Hipertensão Arterial. SBM-Seminários Brasileiros em Medicina, São Paulo, v. 1, n. 1, p. 14-15, 2006. 